\title{
Fast Texture Synthesis And Image Completion Method
}

\author{
Mohiy M. Hadhoud \\ Menoufia University, \\ Faculty of Computers and \\ Information, Information \\ Technology Dept., Egypt, \\ mmhadhoud@yahoo.com
}

\author{
K. A. Mostafa, \\ Menoufia University, \\ Faculty of Computers and \\ Information, Information \\ Technology Dept., Egypt \\ kaaly2006@,yahoo.com
}

\author{
Sameh. Z. Shenoda \\ Menoufia University, \\ Faculty of Computers and \\ Information, Information \\ Technology Dept., Egypt \\ sameh_37@hotmail.com
}

\begin{abstract}
So in this paper, we present a fast texture synthesis and image completion method of natural scenery, where the removal of a foreground object creates a hole in the image. A fast and good quality resulted from the proposed method compaired with others are introduced. Finally the computation time of our method is decreasing from (days, hours and minutes for compaired methods) to less than one second.
\end{abstract}

Keywords-: image restoration, inpainting, texture synthesis and image completion.

\section{Introduction}

Traditionally, skilled artists have performed the restoration of image manually. now digital techniques are used for automatic images restoration to modify the damage area in a non-detectable way for an observer not familiar with the original images.

The restoration can be done by using three related approaches, variational image inpainting, texture synthesis and image completion, whereas the meaning of the first approach is restoring the missed and damaged parts of image in a way that the observer who doesn't know the original image can't detect the difference between the original and the restored image by filling unknown area on the image by using surrounding structure information [1].

The second approach is classified into two methods, texture synthesis and constrained texture synthesis. The texture synthesis method is an input sample of a given texture, and the goal is to produce more of that texture, but the constrained texture synthesis method is filling unknown area on the image by using surrounding texture information. Texture synthesis approaches could be employed to restore digitized photographs especially if a damaged area needs to be filled with some pattern or structure. Texture synthesis does a good job if the sample area in the same image is large enough. However texture synthesis usually fails if the area to be reconstructed contains an additional color or intensity gradient [2].

In recent years, for two dimensional digital images, the third approach combines both texture synthesis and inpainting approaches to restore large gaps in the image by filling unknown area on the image by using both texture and structure information .

The difference between the three related filed is the size of the region or hole to be restored and the type of data to fill the region. And the common requirements of all the three related approaches are the region to be restored manually selected by the user, because there is no mathematical equation capable of detecting or knowing the region to be filled without taking desired area $[3,4]$.

There are many applications for each one of the three related approaches: restoration of photographs, films, removal of occlusions such as text, subtitle, logos, stamps and scratches are applied by the first. 3D covering and hole filling are applied by the second. finally removing unwanted objects from images and filling-in the image blocks that are lost in transmission are applied by the third $[1,2]$.

In this paper we proposed a contribution for image completion and texture synthesis applications, where we introduce a fast method for synthesising texture and filling holes caused by removing object without any search or compution compaired to any related work.

This paper is organized as follows. Section 2 gives an overview on the previous texture synthesis and image completion methods. Section 3 presents the proposed algorithm. section 4 illustrate examples of the tested results. Finally the conclusion is drawn in section 5.

\section{Previous work}

From the later section, its clear that the texture synthesis and image completion are important approaches for restoration of large damaged area. So, in the following section we introduce the most related methods used in these approaches. 
Efros and Leung [5] pioneered a nonparametric approach for producing texture from an initial seed instead of applying filters to the sample texture, the model copied the pixels from the sample image itself in synthesis they modeled the texture as a markov random field (MRF). This model produces the new synthesis pixels from a square window around that pixel by indicating the probability distribution of brightness values for a pixel given the brightness values of its spatial neighborhood. The size of the window is a free parameter for the user. This method has few problems such as slipping in the wrong part of search space and growing garbage.

Wei and Levoy [6] extended to Efros and Leung using multi-resolution neighborhood search to reduce the computation time. This algorithm is based on tree structured vector quantization. Texture is synthesized in a coarse to fine manner by using search for the best neighborhood match and multi level resolution pyramid. This method reduces the quality of the result but decreases the computational time.

Drori et al [7] proposed a fragment based algorithm for image completion that could preserve both structure and texture, this method iteratively fills the missing region from the remaining image as the training set using the principle of self similarity, a confidence map is used to determine which pixels have more surrounding information available. The completion starts from more confident pixels, and proceeds in a multi scale fashion from coarse to fine. In each step, a similar image fragment is found and copied to current unknown location, until image is completed. Most results of this method are good in quality but very slow in time more than hour to complete the image .

Peng Tang [2] proposed a non parametric technique based on a markov random field model. The non parametric sampling procedure for filling the image utilizes a series of binary mask Gaussian pyramid level texture to synthesized the texture in a coarse to fine order. The sampling process randomly and uniquely chooses a pixel from the initial synthesis guess level

Criminisi et al [8] proposed exemplar based method similar to fragment based completion in [8] but simple and fast. It selects the patch located at the gap boundary and search for similar patch in the known region in the whole image with special priority to the isophotes (line of equal gray value) so as to preserve the linear structure of filling then copy the matched patch or block from the known region to fill the gap.

Komodakis and Tziritas [9] proposed a new exemplar based method for image completion and texture synthesis as a discrete global optimization problem with a well defined objective function. The optimal solution founded by priorityBP algorithm that which carries 2 major improvements over standard belief propagation first "label pruning" that accelerate the process by allowing less number of source locations that can be copied to more confident point. Second "priority based message scheduling" that also speeds up by giving high priority to belief propagation from more confident points. This method presented better result and the computation lasted only few seconds up to 2 minutes for an image .

Norihiko, Tomokazu and Naokazu [10] proposed new approach that is different from conventional ones in the objective function. This method extended the conventional objective function that based on pattern similarity SSD (sum of square difference) by allowing brightness change of sample and introducing spatial locality. This extension improves the quality but increases the computational time.

The work done by drori[7] and Criminisi [8] comes closest to the work of our paper. The missing area is iteratively filled using the remaining image but our paper remove the search process of the whole image and find the appropriate texture from the principles of a strong horizontal orientation of texture/color distribution and the two sides scanning.

\section{Proposed Method}

In figure1, the block diagram of the image restoration with any one of the three related approach are shown, the difference between all the image inpainting, texture synthesis and image completion algorithms are the method of filling. All the three related filed must select the region to be filled manually and initializing the selected region by clearing its color. the time of filling is linear to the size of region not the size of image.

Now we come to the block of the proposed filling method. Our proposed filling method is based on two main principles in built it. The first is a strong horizontal orientation and the second is a two side scanning method. By using these principles, we can decrease the time of filling from a few days to less than one second by canceling the search process which takes more time in all previous techniques. Also, by using the same principles for proposed method the quality of restored image increase by filling the region from the two side. 


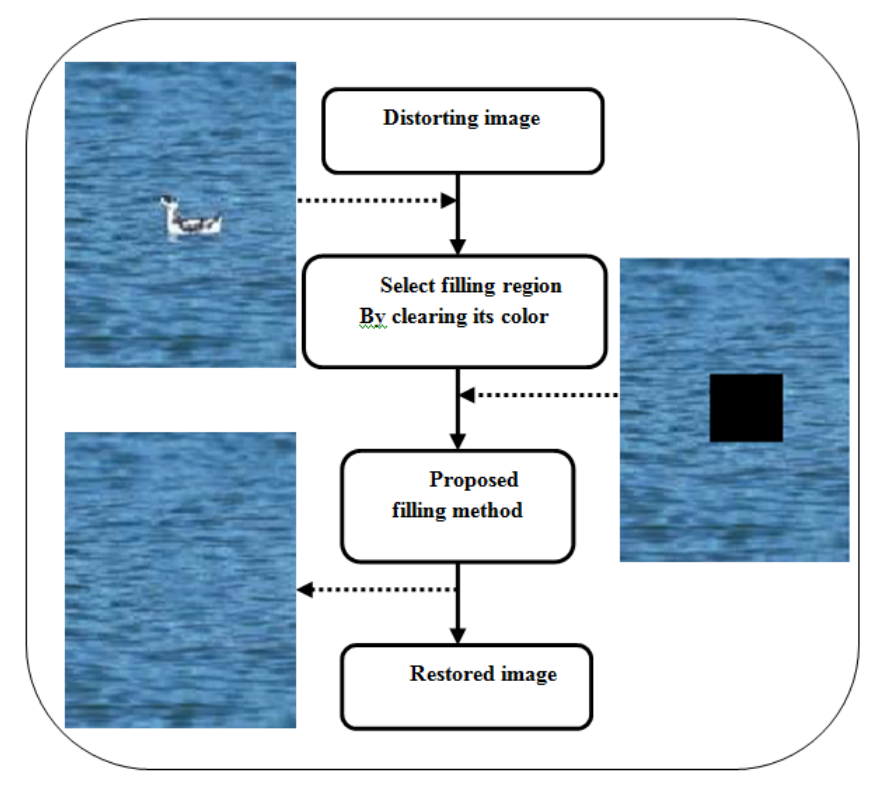

Figure 1 image restoration block diagram

From the natural appearance of the image it is observed that the first principle in images with natural scenery have a strong horizontal orientation of texture/color distribution. We have tried to take the advantage of this fact in our proposed algorithm to fill the missing region .

The assumption of horizontal orientation in natural images is also supported by the Fourier transform of such image, Fourier transform of such image is shown in Figure 2. we observed from the transform, that there is a distinct vertical line at the center. This indicates that the color/texture in the image is horizontally oriented.

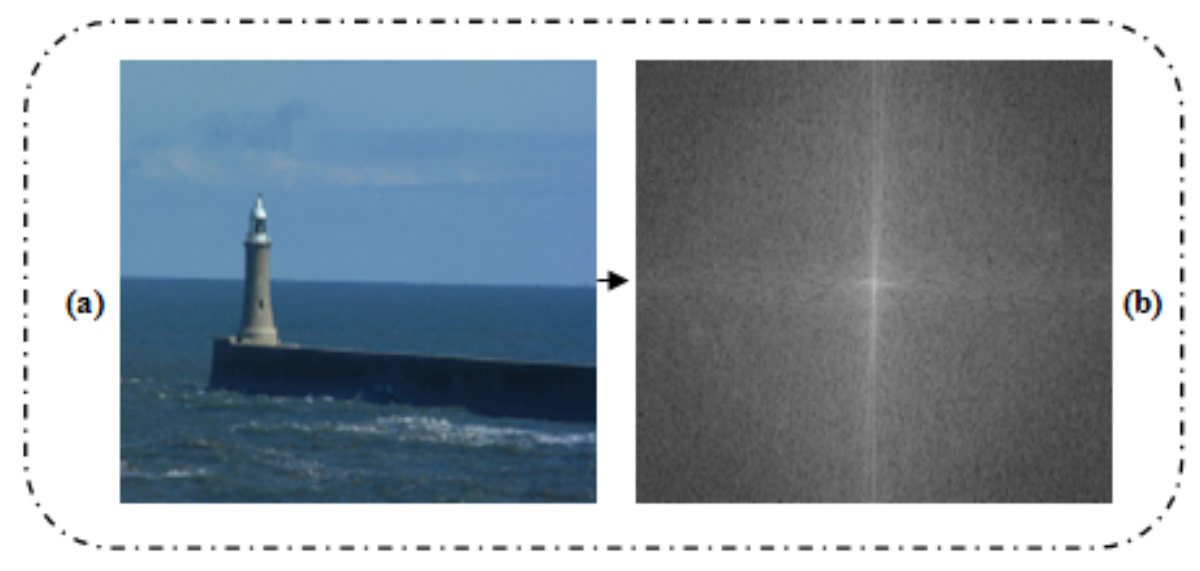

Figure 2 Fourier transform of natural image

a) RGB Image, b) Fourier transform of RGB

We use the image as our training set to complete the image from the horizontal neighborhoods without any search computations.

From the natural appearance of the image, it is also observed the second principle in images with natural scenery or texture image. We noted before removing any object or part from image that the data in the left and right the object is the acceptable data or background that if we fill the region with this data the image appears as well as normal as we show in figure 3 . 


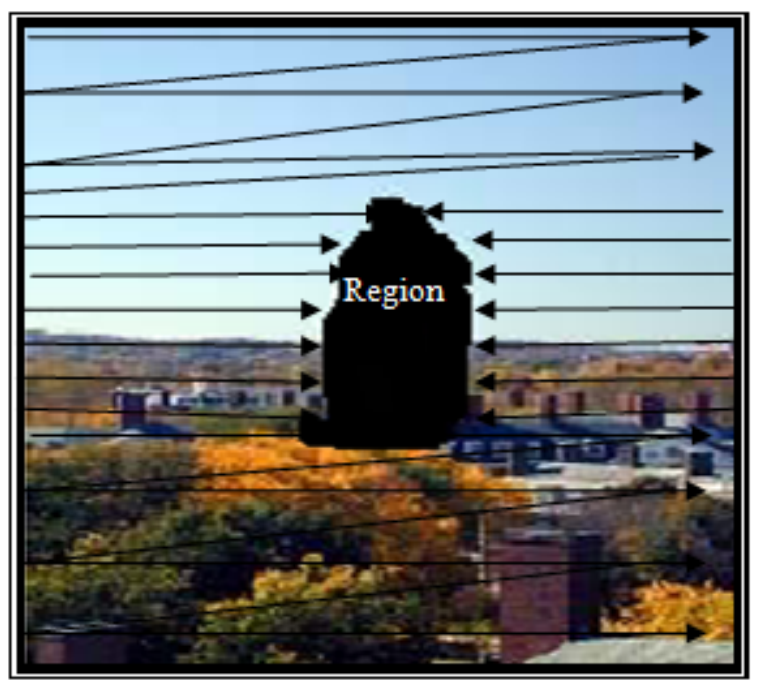

Figure 3 Proposed scanning and filling method

The filling with two side as shown above is better than one side because two reasons. The first reason is scanning and filling with two side decreases the time. The second reason is with one side may fill region with unacceptable data if the object located in non homogeneous texture and spans different homogeneous region. Because of these reasons the algorithm maintains figural similarity by filling the left half of the region with the left side of the known image data and filling the right half of the region with the right part of the known image data .

After explaining the two main principles of our proposed work, now we explain the detailed algorithm.

Instead of explaining the algorithm in a text form we show in figure 4 the detailed flowchart of the proposed method explaining the algorithm step by step in a graph form.

The flowchart is shown in brief. The algorithm makes a raster scan until reaching the first damage pixel, after reaching the first damage pixel the algorithm starts the two side scanning and filling until damage region complete. The detailed steps are shown in the flowchart.

\subsection{Concave problem}

We fill-up the unknown region with pixels from the source image. In most of the images the removed foreground portion is one single area with a convex shape. Considering a grid row, the unknown region is continuous in such shapes. In some images, the shape of the missing region could be concave. This may cause a small part of the source image to be available between two limbs of the concave region. Due to lack of sufficient number of pixels along the horizontal row, the algorithm would attempt to select some pixels from the missing area itself (self-replacement), causing figural discontinuities in the missing area along one or more rows.

When we come across a row where there are such discontinuities, the result of filling for the source pixels would recover some pixels that are themselves unknown. This means that the pixel in the missing area is being filled in by another pixel from the missing area itself. As we proceed further, the error gets propagated along the row.

The algorithm uses the following method to handle such images with the concave region, when the algorithm fills the row between the two saved region points pixel by pixel it is checked first before filling if the pixel is known between the two limbs of the concave region the algorithm skips this pixel with no change or if the pixel is region pixel (unknown) in one of the two limbs the algorithm continues as the same way as explained later.

\section{Dissection of Results}

The algorithm is applied with $\mathrm{C}++$ Builder on $1.7 \mathrm{GHz}$ PC processor and 256 of RAM, after experimented the results they are produced in less than one second. To demonstrate the effectiveness of our method we have applied a comparison between our method and latest four conventional methods peng [2], Drori [7], Komodakis [9] and Norihiko [10] as shown in the next comparison tables. 


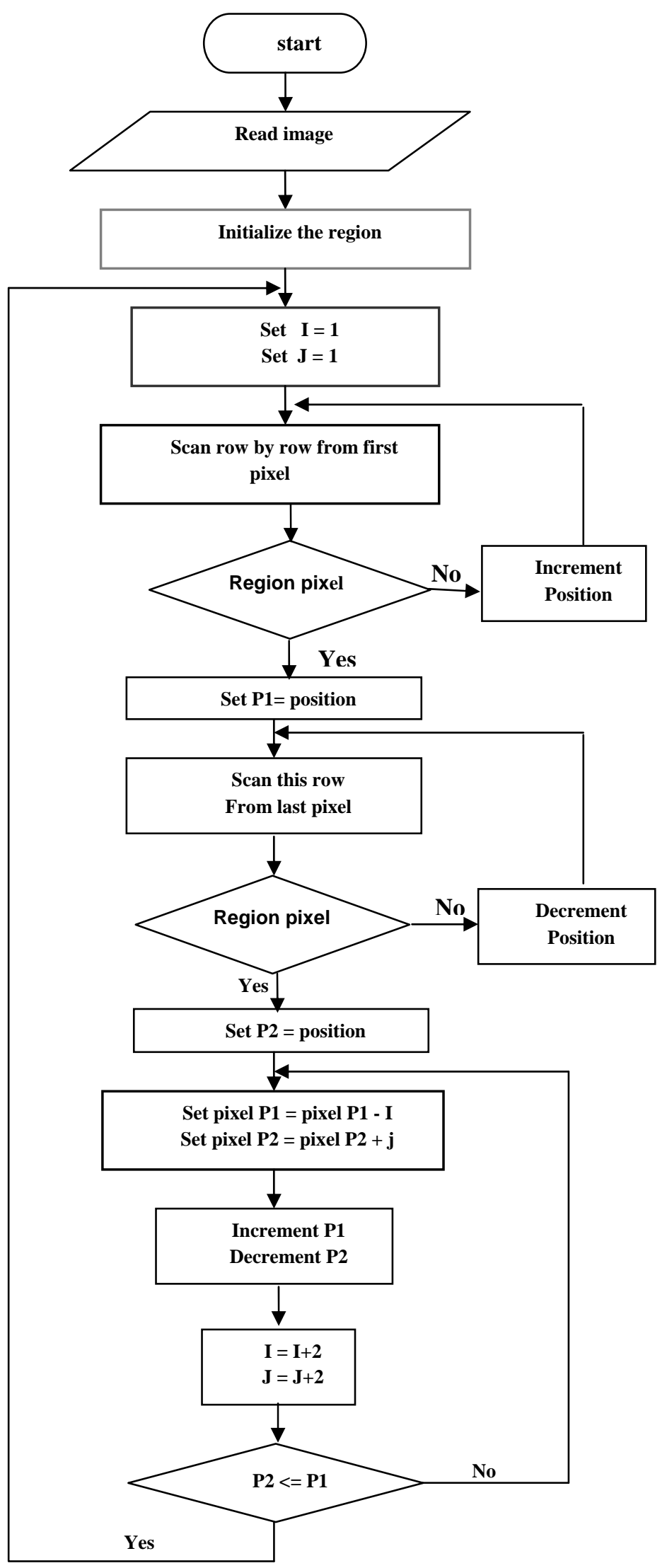

Figure 4 Proposed scanning and filling method 
Mohiy M. Hadhoud, K. A. Mostafa, Sameh. Z. Shenoda

Table 1 processing time of proposed and peng methods

\begin{tabular}{|l|c|c|}
\hline \multicolumn{1}{|c|}{ image } & $\begin{array}{c}\text { Proposed } \\
\text { By CPU speed } \\
\mathbf{1 . 7} \mathbf{~ G H z}\end{array}$ & $\begin{array}{c}\text { Ref.[2] } \\
\text { By CPU speed } \\
\mathbf{2 . 0} \mathbf{~ G H z}\end{array}$ \\
\hline Fig 5 Tower & Less than 1 sec & days \\
\hline Fig 5 Horse & Less than 1 sec & days \\
\hline Fig 5 Texture & Less than 1 sec & days \\
\hline
\end{tabular}

Table 2 processing time of proposed and Drori methods

\begin{tabular}{|l|l|c|}
\hline \multicolumn{1}{|c|}{ image } & $\begin{array}{c}\text { Proposed } \\
\text { By CPU speed } \\
\mathbf{1 . 7} \mathbf{~ G H z}\end{array}$ & $\begin{array}{c}\text { Ref. [7] } \\
\text { By CPU speed } \\
\mathbf{2 . 4} \mathbf{~ G H z}\end{array}$ \\
\hline Fig 6 Dolphin & Less than 1 sec & $184 \mathrm{sec}$ \\
\hline Fig 6 Cup & Less than 1 sec & $149 \mathrm{sec}$ \\
\hline Fig 6 Elephant & Less than 1 sec & 83 minutes \\
\hline
\end{tabular}

Table 3 processing time of proposed and Komodakis methods

\begin{tabular}{|l|c|c|}
\hline \multicolumn{1}{|c|}{ image } & $\begin{array}{c}\text { Proposed } \\
\text { By CPU speed } \\
\mathbf{1 . 7} \mathbf{~ G H z}\end{array}$ & $\begin{array}{c}\text { Ref. [9] } \\
\text { By CPU speed } \\
\mathbf{2 . 4} \mathbf{~ G H z}\end{array}$ \\
\hline Fig 7Golf & Less than 1 sec & up to 2 minutes \\
\hline Fig 7 Giraffe & Less than 1 sec & up to 2 minutes \\
\hline Fig 7 Baseball & Less than 1 sec & up to 2 minutes \\
\hline
\end{tabular}

Table 4 processing time of proposed and Norihiko methods

\begin{tabular}{|l|c|c|}
\hline \multicolumn{1}{|c|}{ image } & $\begin{array}{c}\text { Proposed } \\
\text { By CPU } \\
\text { speed } \\
\mathbf{1 . 7} \text { GHz }\end{array}$ & $\begin{array}{c}\text { Ref. [10] } \\
\text { By CPU } \\
\text { speed } \\
\mathbf{3 . 2 ~ G H z}\end{array}$ \\
\hline Fig 8 Man & Less than 1 sec & 8.45 hours \\
\hline Fig 8 Man \& Boy & Less than 1 sec & 18.59 hours \\
\hline Fig 8 Mountain & Less than 1 sec & 5 hours \\
\hline
\end{tabular}

Figure 5 and table 1 illustrate the comparison between the proposed method and Ref.[2] depend on three different images. In the first image in this figure the object is located in non homogeneous texture and spans different homogeneous region. In the second image the object is located in one homogeneous region. In the third image it's a stochastic texture image. our algorithm gives a very good quality with very short time compaired with Ref. [2] in the same image and size.

Figure 6 and table 2 show another images comparison between the proposed method and Ref.[8] . All of the images have a concave region and our algorithm gives a good result in that type also with fewest time. All result produced in [7] range from 120 to 419 seconds for 192 by 128 images and range from 83 to 158 minutes for 384 by 256 images .

Figure 7 shows the proposed method results and the method of Ref [9] with the same image and size. This method is the closest method to our method in time as shown in table 3.

Figure 8 illustrate the proposed method results and the method of Ref [10] with the same image and size, this method give a better quality than our method in the first image but our method gives a good quality than [10] in the second and third image , table 4 show a comparison in processing time.

At the end our algorithm work well in image completion and texture synthesis with very good result in quality and smallest time than other methods.

\section{Conclusions}

We have offered a fast image completion and texture synthesis algorithm with a very good quality than other methods, and with no search computational time to give smallest method in time. 


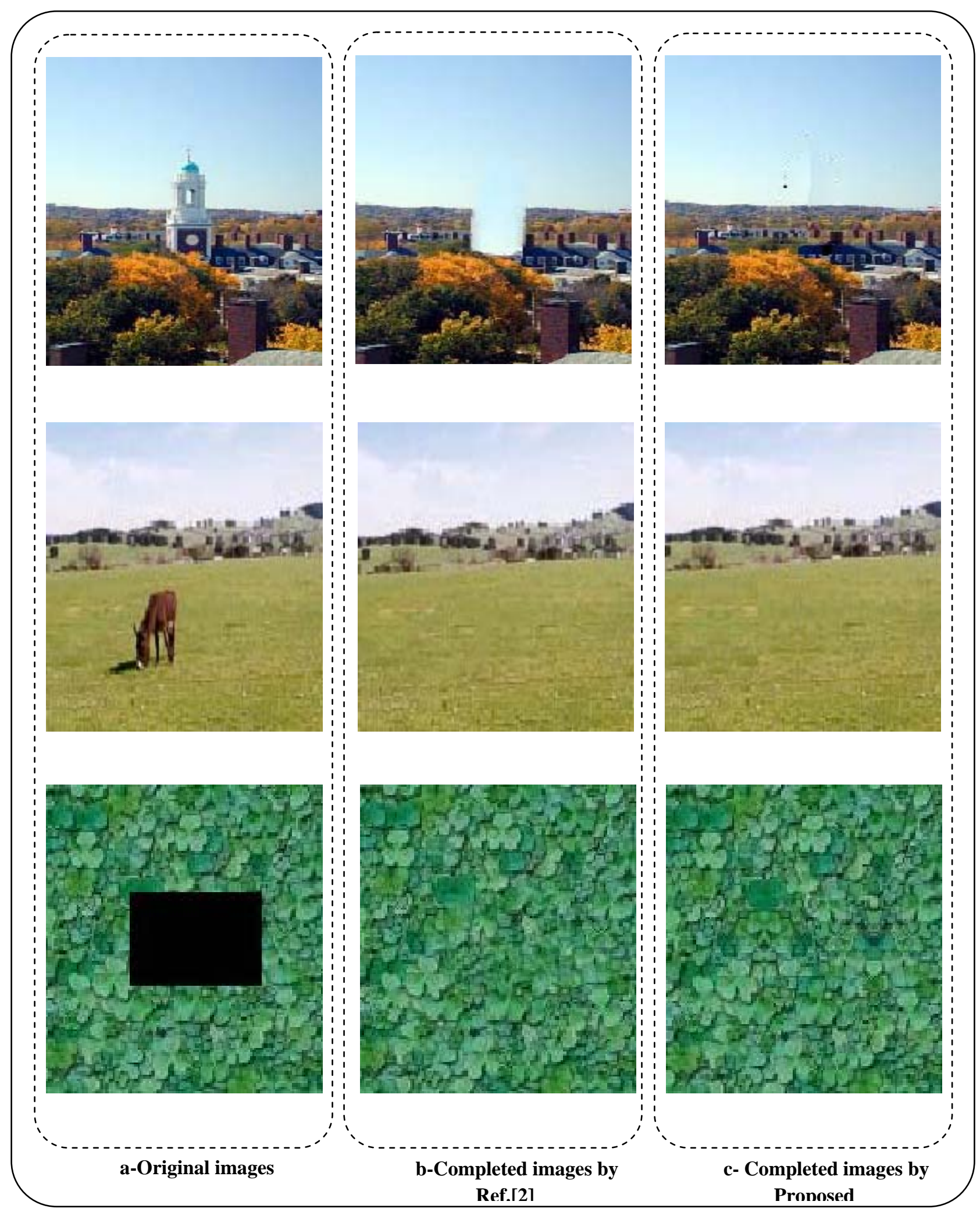

Figure 5 Comparison result of Proposed method with Ref.[2] 
Mohiy M. Hadhoud, K. A. Mostafa, Sameh. Z. Shenoda

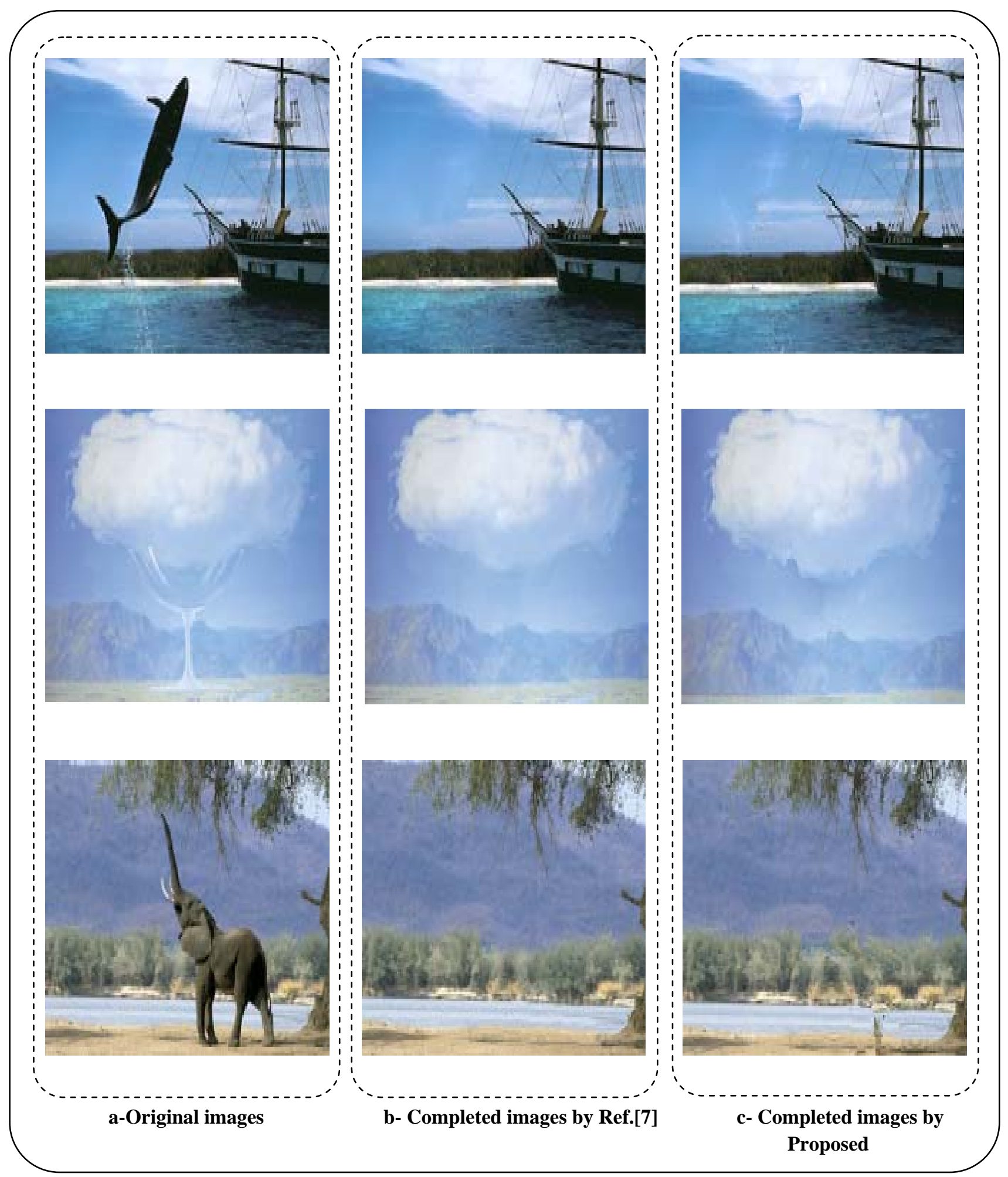

Figure 6 Comparison result of Proposed method with Ref.[7] 


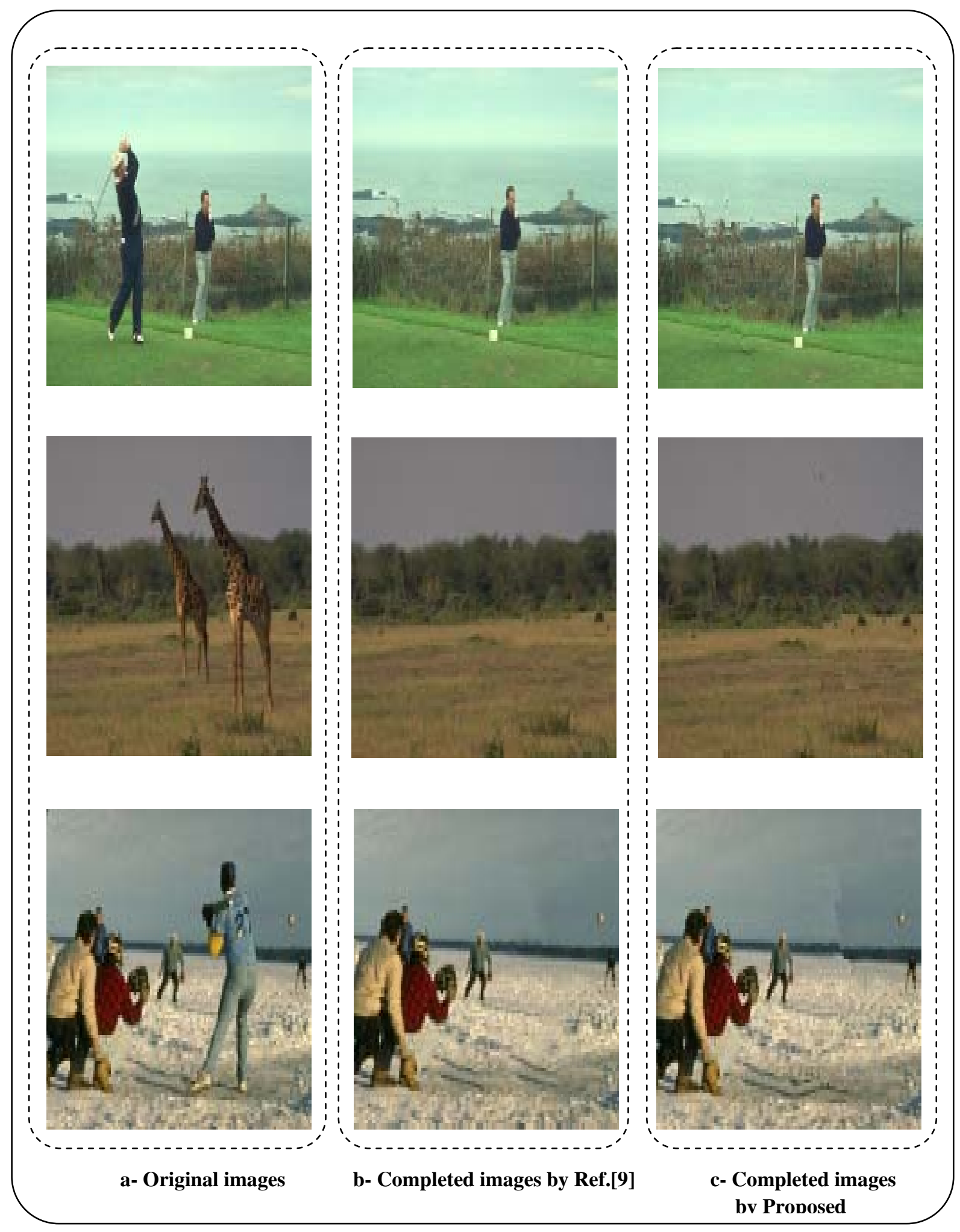

Figure 7 Comparison result of Proposed method with Ref.[9] 
Mohiy M. Hadhoud, K. A. Mostafa, Sameh. Z. Shenoda

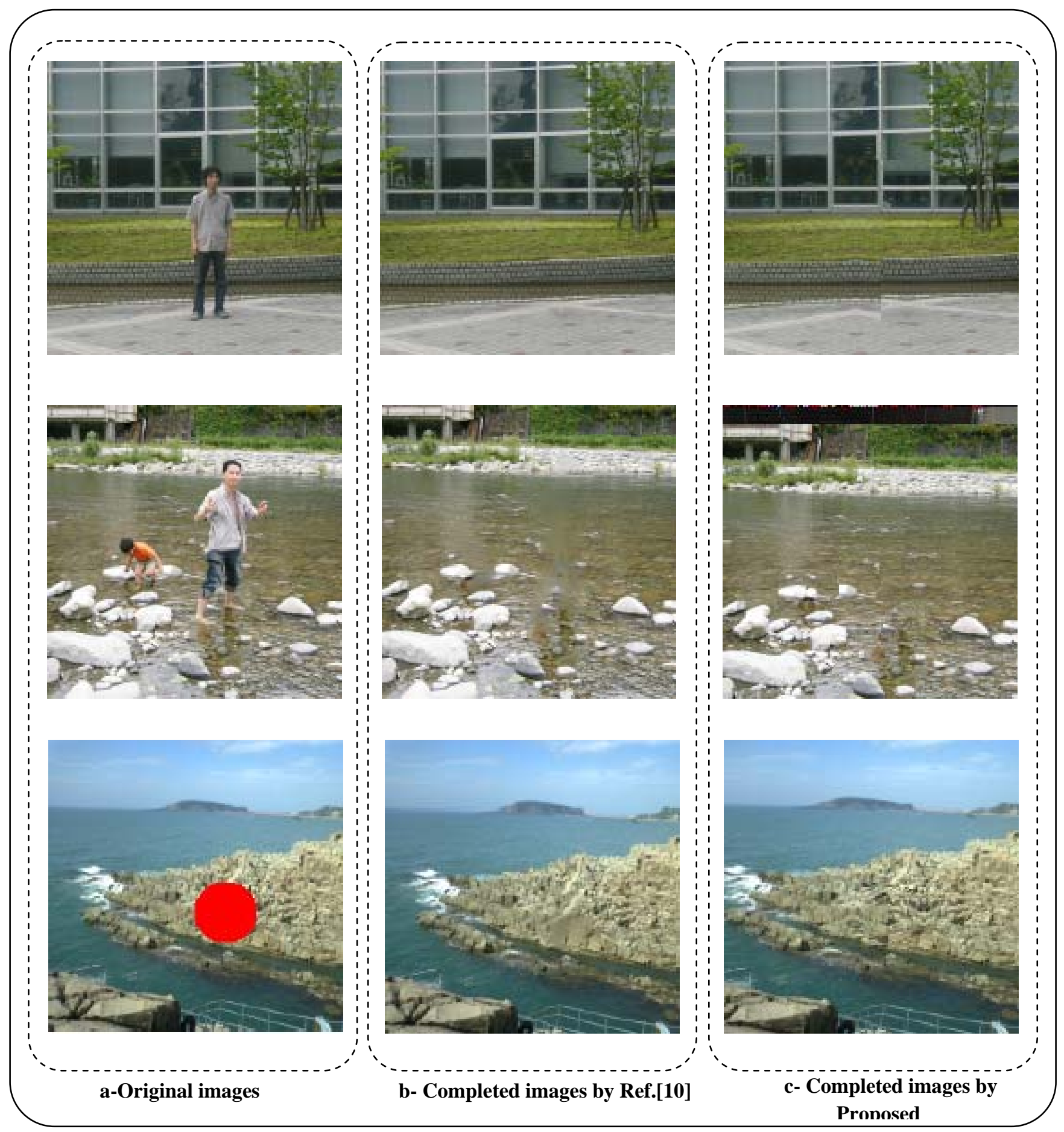

Figure 8 Comparison result of Proposed method with Ref.[10] 


\section{References}

[1] A.telea " An image inpainting technique based on the fast marching method" journal of graphics tools, vol. 9, no. 1 , acm press 2004.

[2] Peng Tang "Application of non parametric texture synthesis to image inpainting" thesis, Albuquerue, New Mexico 2004.

[3] S. Zelinka and M. Garland "jump map based interactive texture synthesis " ACM Trans. Graph.,vol. 23, no 4, pp 930-962, 2004.

[4] Işık Barış Fidaner "A Survey on variational image inpainting,texture synthesis and image completion " Bo_gazic,i University 2007.

[5] A. Efros and T. Leung, "Texture synthesis by non-parametric sampling" in ICCV (2), pp. 1033-1038. 1999

[6] L.-W. Wey and M. Levoy, "Fast texture synthesis using tree-structured vector quantization," in Siggraph 2000, Computer Graphics Proceedings ,K. Akeley, Ed. ACM Press / ACM SIGGRAPH / Addison Wesley Longman, pp. 479-488. 2000.

[7] I. Drori, D. Cohen-Or, and H. Yeshurun, "Fragment-based image completion" in SIGGRAPH ACM SIGGRAPH 2003 Papers. New York, NY, USA: ACM Press, pp. 303-312, 2003.

[8] P. P. A. Criminisi and K. Toyama, "Region filling and object removal by exemplar-based inpainting," IEEE Trans. Image Processing, vol. 13, no. 9, pp. 1200-1212, 2004.

[9] N. Komodakis, "Image completion using global optimization" in CVPR '06: Proceedings of the 2006 IEEE Computer Society Conference on Computer Vision and Pattern Recognition. Washington DC,USA IEEE Computer Society, pp. 442-452, 2006.

[10] Norihiko Kawai, Tomokzu and Naokazu "image inpainting considering brightness change and spatial locality of textures" graduate school of information science, nara institute of science and technology,japan 2008 . 